\title{
Activité acoustique et loi de dispersion : le quartz et le chlorate de sodium
}

\author{
C. Joffrin $(*)$, B. Dorner $\left({ }^{* *}\right)$ et J. Joffrin $(* *)$ \\ (*) Laboratoire Léon-Brillouin, CEA Orme des Merisiers, B.P. № 2, 91190 Gif-sur-Yvette, France \\ et Institut Laue-Langevin, Avenue des Martyrs, 156 X, 38042 Grenoble Cedex, France \\ (**) Institut Laue-Langevin, Avenue des Martyrs, 156 X, 38042 Grenoble Cedex, France
}

(Reçu le 22 mai 1980, accepté le 26 juin 1980)

\begin{abstract}
Résumé. - Par diffusion inélastique de neutrons, on a mesuré le pouvoir rotatoire acoustique naturel de deux cristaux de symétrie différente : le quartz et le chlorate de sodium.
\end{abstract}

\begin{abstract}
Acoustical activity has been measured in two different crystals (quartz and $\mathrm{ClO}_{3} \mathrm{Na}$ ) by neutrons inelastic scattering.
\end{abstract}

1. Introduction. - Le phénomène de l'activité acoustique naturelle des cristaux est prouvé; il consiste en une rotation du plan de polarisation d'ondes transversales lorsque ces dernières se propagent dans des directions bien définies d'un cristal auquel manquent certains éléments de symétrie. Cette rotation résulte de la décomposition en deux ondes polarisées circulairement en sens inverse dont les vitesses de propagation sont différentes. Cet effet traduit une levée de dégénérescence due à la non-localité des équations de matière, c'est-à-dire à la dispersion spatiale de la constante élastique si l'on garde le langage de l'élasticité classique. L'étude théorique de ce phénomène $a$ été faite [1]; en particulier, les classes de symétrie où l'activité acoustique naturelle peut être observée ont été inventoriées : elle apparaît dans les cristaux qui possèdent un axe d'ordre 3 ou supérieur et qui n'ont ni plan de symétrie ni axe d'inversion.

Des considérations analogues s'appliquent aux ondes optiques. Le pouvoir rotatoire optique se manifeste le long des axes optiques des cristaux cubiques uniaxes dits énantiomorphes ou des cristaux hexagonaux. Dans les éléments de symétrie de ces cristaux ne figurent ni miroir ni inversion. C'est la dispersion spatiale de la constante diélectrique qui est alors à l'origine du phénomène observé.

Le pouvoir rotatoire acoustique naturel du quartz $\alpha$ le long de l'axe [001] ou plutôt la levée de dégénérescence des modes transversaux a été mise en évidence d'abord par des expériences de neutrons [2] ; mais les mesures étaient trop imprécises pour en permettre une mesure quantitative et d'ailleurs, ce n'était pas leur but. Cet effet n'a pu être observé que par une étude directe de la rotation du plan de polarisation d'une onde transversale par une technique acoustique standard [3] et par effet Brillouin [4]. Les résultats de ces deux types d'approche sont compatibles mais peu précis.

Dans cet article, nous décrivons les résultats obtenus par diffraction inélastique des neutrons en observant la loi de dispersion des modes transversaux pour deux composés : le quartz $\alpha$, (symétrie hexagonale) dans la direction $z$ [001] et le chlorate de sodium, (symétrie cubique) dans les directions [100] et [111].

2. Méthode expérimentale. - Les courbes de dispersion des phonons acoustiques ont été mesurées sur le spectromètre à 3 axes IN 2 installé sur une source thermique du réacteur à haut flux de l'Institut LaueLangevin à Grenoble. Le faisceau monochromatique incident est obtenu par l'intermédiaire d'un double monochromateur. On a choisi de travailler à vecteur d'onde incident constant $k_{\mathrm{I}}=2,662 \AA^{-1}$; dans cette situation, on peut éliminer efficacement la contamination des spectres observés par des réflexions d'ordre supérieur en plaçant devant l'échantillon un filtre de graphite pyrolytique.

La largeur des pics de Bragg est uniquement due à la fonction d'appareil et non à la mosaïcité $\left(n<5^{\prime}\right)$ des deux cristaux étudiés. Les spectres obtenus ont été traités à l'aide d'un programme qui, en comparant les phonons à une gaussienne permettait d'obtenir à partir d'un groupe donné la position, la largeur à mihauteur et l'intensité au sommet de chacun d'eux par l'intermédiaire d'un calcul de moindres carrés (Fig. 1) [5]. Un facteur de normalisation prend en compte les 


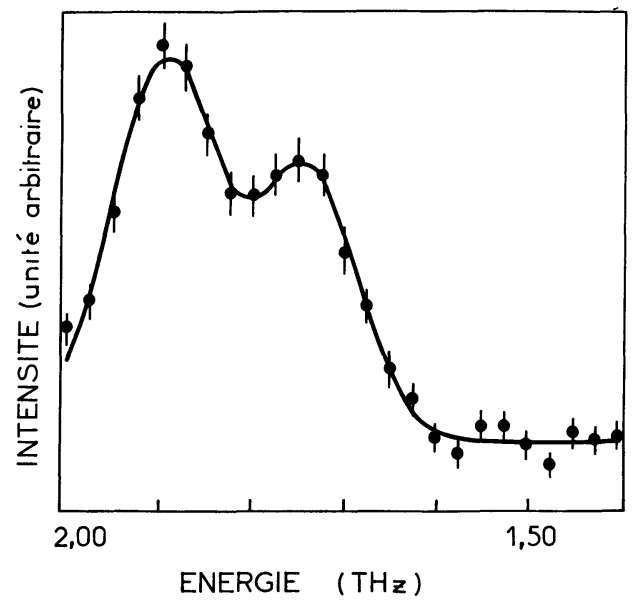

Fig. 1. - Spectre recalculé comme somme de deux gaussiennes ; les énergies des maxima sont ajustées au spectre expérimental par un calcul de moindres carrés et corrigées par la normalisation de la résolution; elles sont $1,744 \mathrm{THz}$ et $1,901 \mathrm{THz}$. Spectre mesuré pour $q_{3}=-0,35$ dans la zone de Brillouin (033) dans le cas de $\mathrm{ClO}_{3} \mathrm{Na}$.

[Recalculated spectrum as sum of two gaussian lines; the energies of the maxima are adjusted to the experimental spectrum by a least square calculation and corrected by normalization of the resolution; they are $1.744 \mathrm{THz}$ and $1.901 \mathrm{THz}$. Spectrum obtained for $q_{3}=-0.35$ in the (033) Brillouin zone for $\mathrm{ClO}_{3} \mathrm{Na}$.]

caractéristiques de l'appareil. Dans le cas du quartz, l'analyse a été compliquée par le fait que le mode longitudinal était faiblement visible. La fréquence de ce mode a été mesurée dans une autre configuration et incluse dans l'analyse sous forme d'une troisième gaussienne dont la position et la largeur étaient supposées connues.

3. Le quartz : $\mathrm{SiO}_{2}$. - Les formes lévogyre et dextrogyre de la variété $\alpha$ du quartz appartiennent aux groupes d'espace trigonaux

$$
\begin{array}{llll}
\mathrm{D}_{3}^{4} & \left(\mathrm{P}_{3}\right. & 2 & 1) \\
\mathrm{D}_{3}^{6} & \left(\mathrm{P}_{2}\right. & 2 & 1)
\end{array}
$$

Les éléments de symétrie contiennent donc un axe d'ordre $3(\mathrm{OZ})$ et perpendiculairement 3 axes d'ordre 2 qui se correspondent dans la rotation d'ordre 3 autour de $\mathrm{OZ}$.

Les paramètres de la cellule élémentaire sont :

$$
\begin{aligned}
& a=4,913 \AA \\
& c=5,404 \AA(\mathrm{OZ}) .
\end{aligned}
$$

Dans la direction $\mathrm{OZ}$ (direction cristallographique 001) et dans la limite de l'élasticité classique, on peut montrer que deux modes acoustiques peuvent se propager : un mode longitudinal pur et un mode transverse pur doublement dégénéré. Les vitesses de ces ondes (pente de la courbe de dispersion en centre de zone) peuvent se calculer à partir des constantes élastiques [8].

La théorie des groupes montre cependant que les deux modes transverses correspondent à deux repré- sentations irréductibles distinctes pour un vecteur d'onde $q$ non nul et lorsqu'on prend en compte la dispersion spatiale de la constante élastique, ces deux modes transverses ne sont plus dégénérés. Après analyse de Fourier, la constante élastique s'écrit :

$$
\begin{gathered}
C_{h j}(\omega, q)=C_{h j}(\omega)+i d_{h j, k}(\omega) q_{k}+\cdots \\
h, j=1 \ldots 6 \\
k=1 \ldots 3 .
\end{gathered}
$$

La matrice dynamique doit alors être calculée en tenant compte des coefficients $d_{h j, k}$ non nuls dont le nombre dépend de la symétrie du cristal considéré.

Il a été montré $[1,4]$ que le coefficient $d_{54,3}$ avait pour effet de lever la dégénérescence des modes transverses dans la direction $\mathrm{O} Z$ du quartz. La résolution de la matrice de Christoffel permet d'obtenir la vitesse des deux modes transverses résultants :

$$
\frac{\omega_{ \pm}^{2}}{q_{3}^{2}}= \pm v^{2}=\frac{1}{\rho}\left[C_{44} \pm d_{54,3} q_{3}\right]
$$

Ces deux modes ont été très bien caractérisés dans nos expériences (Fig. 2) ainsi que le mode longitudinal dont la courbe de dispersion est pratiquement linéaire dans la région étudiée. La pente à l'origine de ces courbes permet de calculer la vitesse des modes correspondants; elles sont compatibles avec les valeurs

\section{Quartz}

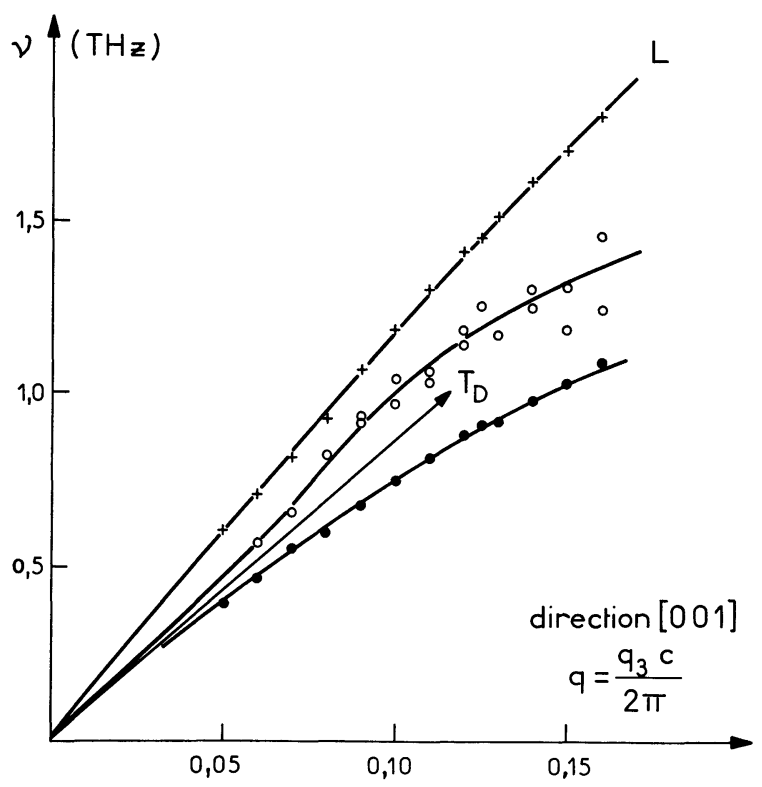

Fig. 2. - Courbes de dispersıon du quartz- $\alpha$ dans la direction $\mathrm{OZ}$ les points portés sont ceux obtenus après traitement numérique des données brutes. Les deux droites $L$ et $T_{D}$ ont des pentes qui correspondent aux vitesses des ondes acoustiques à $q=0$.

[Dispersion curve for $\alpha$-quartz in the $\mathrm{OZ}$ direction; the plotted points are obtained after numerical treatment of the raw data. The two straight lines $L$ and $T_{D}$ have slopes which correspond to speed of acoustical waves at $q=0$.] 
Tableau I.

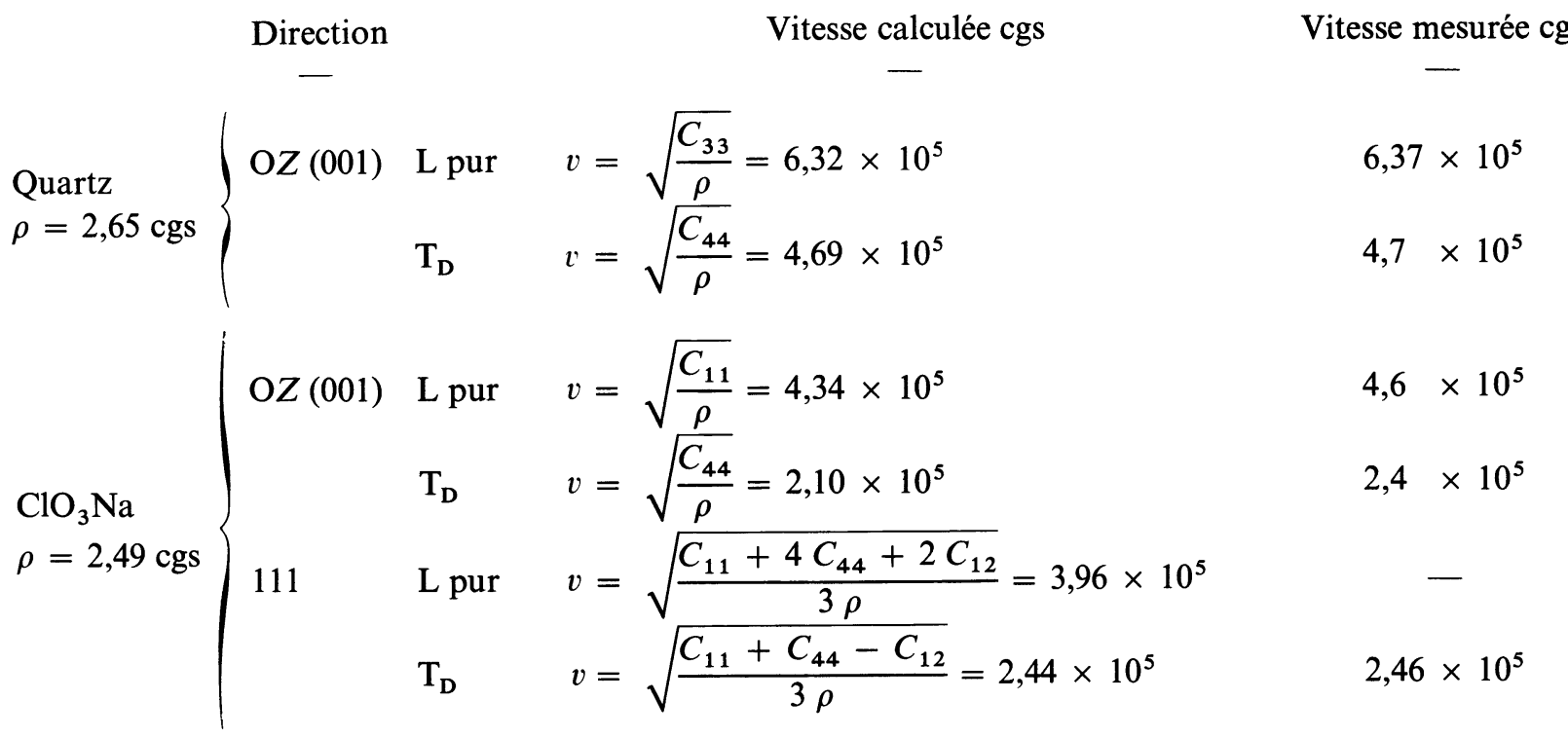

théoriques calculées à partir des constantes élastiques $C_{33}$ et $C_{44}$ (tabl. I).

Lorsqu'on s'éloigne du centre de zone, le mode transverse se dédouble; le coefficient de couplage $d_{54,3}$ peut être calculé à partir de la formule (2) soit

$$
\omega_{ \pm}^{2}=q_{3}^{2} \frac{1}{\rho}\left(C_{44} \pm d_{54,3} q_{3}\right)
$$

on a

$$
\omega_{+}-\omega_{-}=\Delta \omega \simeq \frac{d_{54,3}}{\rho \bar{v}} q_{3}^{2}=\frac{d_{54,3}}{C_{44}} \bar{v} q_{3}^{2}
$$

La différence des fréquences $\Delta \omega$ entre les deux modes transverses doit varier comme $q_{3}^{2}$ dans la mesure où $\Delta \omega \ll \omega$.

La figure 3 montre qu'il en est ainsi pour $q_{3}<1 / 10 q_{\text {Brillouin }}$; à partir de la pente de la droite ainsi obtenue, on peut calculer le rapport

$$
\frac{d_{54,3}}{C_{44}}=(2,8 \pm 0,2) \cdot 10^{-8} \mathrm{~cm} .
$$

On peut également calculer

où

$$
\frac{\Delta v}{\bar{v}}=\frac{d_{54,3}}{C_{44} \bar{v}} \omega
$$

$$
\frac{\Delta v}{\bar{v}}=\frac{2 \pi d_{54,3}}{C_{44} \bar{v}} v=3,7 \times 10^{-13} v(v \mathrm{en} \mathrm{Hz}) .
$$

Le tableau ci-dessous résume les valeurs obtenues pour $\Delta v \sqrt{v}$ pour les différents types de mesures mentionnées plus haut :

\section{Références}
A. S. Pine [3]
J. Joffrin, A. Levelut [4]
Ce travail

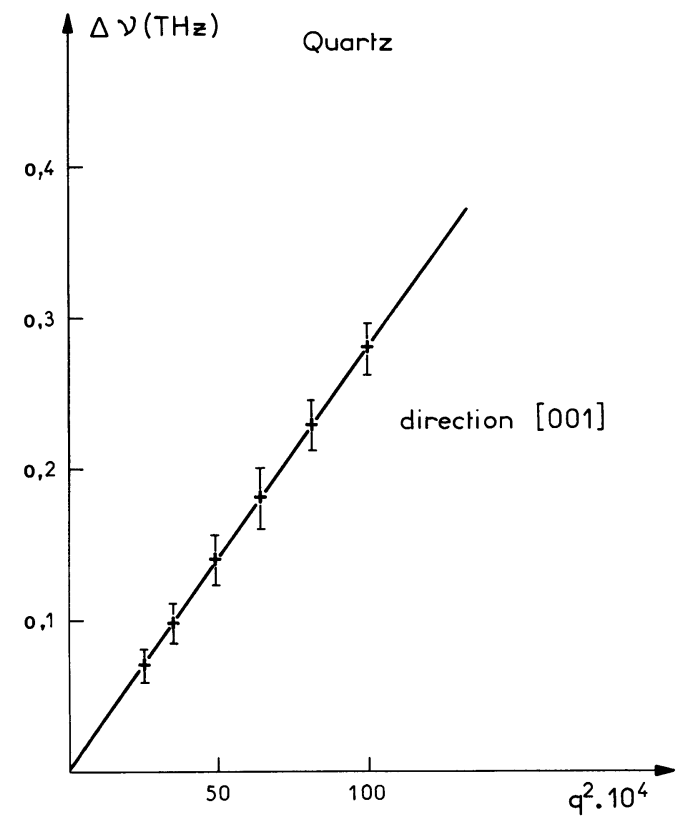

Fig. 3. $-\Delta \omega$ en fonction de $q_{3}^{2}$ en unités de l'espace réciproque pour le quartz. Seules les 6 premières valeurs déduites des courbes de la figure 2 ont été notées ici dans la mesure où, pour $q_{3}>0,1$, la courbure normale des lois de dispersion obscurcit le résultat de la levée de dégénérescence par effet de pouvoir rotatoire acoustique.

[ $\Delta \omega$ as a function of $q_{3}^{2}$ in units of the reciprocal space for quartz. Only the six first values deduced from figure 2 have been used here because, for $q_{3}>0.1$, the standard curvature of the dispersion law masks the effect of lifting of the degeneration by the acoustical rotatory power.]

4. Cas du chlorate de sodium : $\mathrm{NaClO}_{3}$. - Le chlorate de sodium $\left({ }^{1}\right)$ appartient au groupe d'espace cubique P2 3 [9] (groupe ponctuel 23 ). L'intérêt de mesurer le pouvoir rotatoire dans ce cristal vient de ce

( $\left.{ }^{1}\right)$ Un très bel échantillon de ce cristal nous a été prêté par le Professeur Chapelle, Laboratoire de Physique Cristalline, Orsay. 
qu'aucune expérience n'existe dans des cristaux autres que le quartz. Le paramètre de la cellule élémentaire est

$$
a=6,570 \AA \text {; il y a } 4 \text { molécules par mailles [10]; }
$$

le pouvoir rotatoire acoustique peut être observé dans les deux directions [111] et [100] correspondant à des coefficients $d_{h j, k}$ non nuls différents.

Les constantes élastiques sont [7] :

$$
\begin{aligned}
& C_{11}=4,9 \times 10^{11} \text { dyne } \mathrm{cm}^{-2} \\
& C_{44}=1,2 \times 10^{11} \text { dyne }^{-2} \\
& C_{12}=1,4 \times 10^{11} \text { dyne } \mathrm{cm}^{-2} \text {. }
\end{aligned}
$$

Les valeurs observées et calculées des vitesses des ondes acoustiques dans ces deux directions sont comparées dans le tableau I.

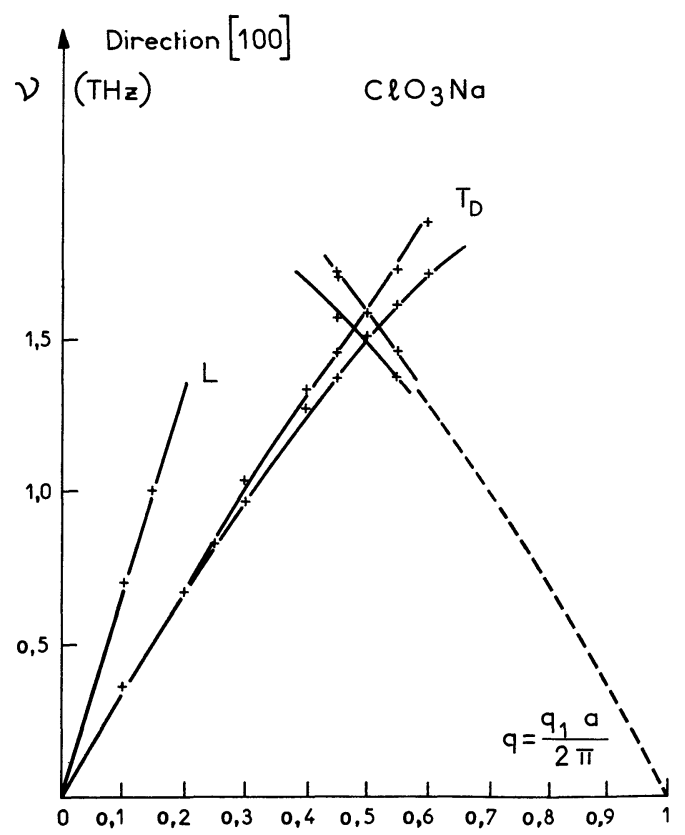

Fig. 4. $-\mathrm{ClO}_{3} \mathrm{Na}$ : courbe de dispersion du mode transverse direction [100]. Au bord de la zone de Brillouin $(q=0,5)$, les courbes de dispersion correspondant à la zone voisine sont visibles également.

$\left[\mathrm{ClO}_{3} \mathrm{Na}:\right.$ dispersion curve of the transverse mode in the [100] direction. At the extremity of the Brillouin zone $(q=0.5)$, the dispersion curves corresponding to the adjacent zone are also visible.]

Dans la direction [100] (voir Fig. 4), le dédoublement est visible; il est dû au coefficient $d_{45,3}$ [1]; il est calculé à partir de la pente de la droite $\Delta \omega=\alpha q_{[100]}^{2}$ (Fig. 6). On trouve $d_{45,3} / C_{44}=(1,5 \pm 0,1) \cdot 10^{-9} \mathrm{~cm}$.

Dans la direction [111] (voir Fig. 5), le coefficient de couplage $d^{\prime}$ responsable de la levée de dégénérescence est une combinaison linéaire de plusieurs $d_{h j, k}$ non nuls [1]

$$
d^{\prime}=\frac{1}{9}\left(3 d_{15,2}-3 d_{16,3}-2 d_{45,3}\right)
$$

l'expression de la vitesse dans cette direction fait inter-

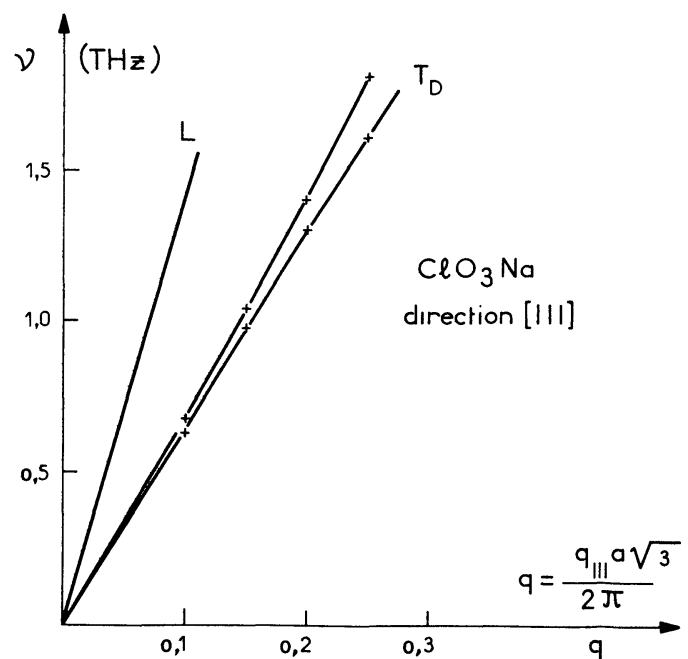

Fig. 5. $-\mathrm{ClO}_{3} \mathrm{Na}$ : courbe de dispersion du mode transverse, direction [111].

$\left[\mathrm{ClO}_{3} \mathrm{Na}\right.$ : dispersion curve of the transverse mode in the [111] direction.]

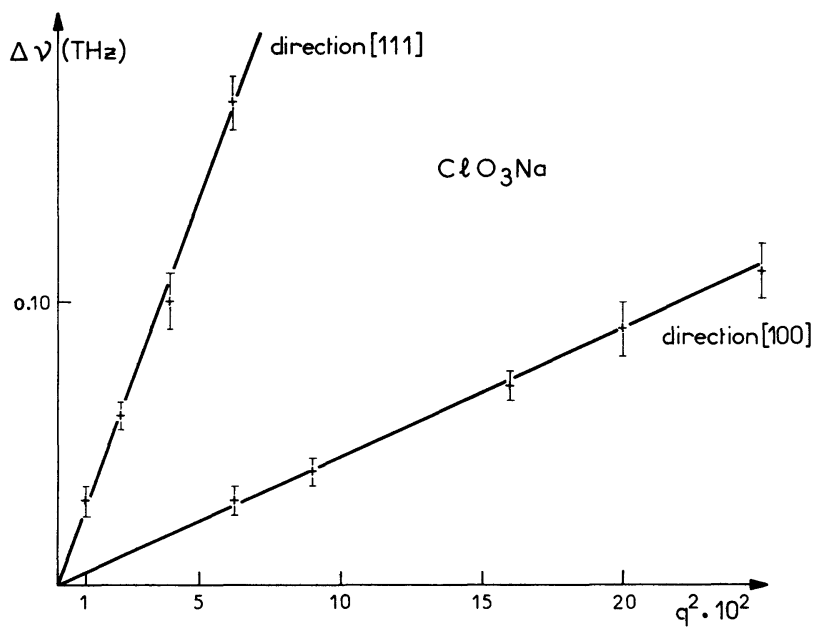

Fig. 6. $-\Delta \omega$ en fonction de $q^{2}$ pour le chlorate de sodium pour les directions [100] et [111].

[ $\Delta \omega$ as a function of $q^{2}$ for sodium chlorate in the [100] and [111] directions.]

venir aussi plusieurs constantes élastiques (tabl. I)

$$
v_{[111]}=\sqrt{\frac{C_{11}+C_{44}-C_{12}}{3 \rho}}=\sqrt{\frac{c^{\prime}}{\rho}} .
$$

A partir de la pente de la droite $\Delta \omega=\alpha^{\prime} q_{[111]}^{2}$ (Fig. 6) on trouve $d^{\prime} / c^{\prime}=(2,3 \pm 0,2) \cdot 10^{-8} \mathrm{~cm}$, ce qui est une valeur comparable à celle trouvée pour le quartz.

Remarquons que dans la direction [100] l'effet observé est d'un ordre de grandeur inférieur à celui noté dans la direction [111]; cela peut être relié au fait que dans le premier cas, il faut lever la dégénérescence de deux nappes de dispersion tangentes alors que dans le deuxième, les deux nappes se coupent dans la direction [111]; la même observation peut être faite dans le cas optique. 
5. Conclusion. - Il existe relativement peu de cristaux dont la symétrie permet d'observer cet effet, et don't les coefficients correspondants ont été mesurés. On a exploré ici le cas du quartz et celui du chlorate de sodium. Il faudrait ajouter les exemples du tellure et du $\mathrm{Bi}_{12} \mathrm{GeO}_{20}$ à propos desquels on aimerait que des expériences soient faites.

Dans la mesure où aucun des modèles relatifs aux courbes de dispersion n'a vraiment pris en compte ce paramètre $d$, ou plutôt, n'a jamais ajusté les constantes de forces élémentaires pour rendre compte de la valeur de ce paramètre $d$, il est difficile de voir comment il modifie les idées que l'on a à l'heure actuelle. Il nous parait cependant utile de souligner que ce paramètre joue le même rôle qu'une constante élastique dans toute construction de modèle de force au niveau atomique.

Une démarche qu'il peut être intéressant de souligner, c'est de voir s'il existe une corrélation entre le pouvoir rotatoire acoustique naturel et le pouvoir rotatoire optique naturel. Ils s'expriment sous des formes très similaires, respectivement :

$$
\begin{aligned}
& \frac{\varphi}{l}=\frac{1}{2} \frac{d}{c} q^{2} \\
& \frac{\varphi}{l}=\frac{1}{2} \frac{g}{\varepsilon} q^{2}
\end{aligned}
$$

où $q$ est le vecteur d'onde, $d$ ou $g$ sont les éléments non nuls du tenseur gyrotropique acoustique (tenseur du $5^{\mathrm{e}}$ ordre) et optique (tenseur $3^{\mathrm{e}}$ ordre), $c$ est la constante élastique et $\varepsilon$ la constante diélectrique. On peut dresser là où c'est possible un tableau comparatif des deux quantités qui caractérisent cet effet. $\mathrm{Ce}$ pouvoir rotatoire spécifique est donné dans le tableau II pour

Tableau II. - Pouvoir rotatoire spécifique en degré/mm pour une longueur d'onde de $0,59 \mu$.

[Specific rotary power in degree per millimeter for a wavelength of $0.59 \mu$.]

$\begin{array}{lcc} & \text { Acoustique } & \text { Optique } \\ & - & - \\ \text { Quartz }_{[001]} & 916 & 21,7[7] \\ \mathrm{ClO}_{3} \mathrm{Na}_{[100]} & 47,5 & 3,1[7][11] \\ \mathrm{ClO}_{3} \mathrm{Na}_{[111]} & 750 & \end{array}$

les mêmes valeurs du vecteur d'onde optique ou acoustique ; il apparaît clairement que le pouvoir rotatoire exprimé en rotation spécifique est plus grand dans le cas acoustique que dans le cas optique, observation qui devrait elle aussi être reliée avec un modèle microscopique.

\section{Bibliographie}

[1] Portigal, D. L., Burstein, E., Phys. Rev. 170 (1968) 673 ; Kluge, G., Scholz, G., Acustica 16 (1965) 60.

[2] Elcombe, M., Proc. Phys. Soc. 91 (1967) 947.

[3] Pine, A., Phys. Rev. B 2 (1970) 2049.

[4] Joffrin, J., Levelut, A., Solid State Commun. 8 (1970) 1573.

[5] Dorner, B., Acta Crystallogr. A 28 (1972) 319.

[6] Saksena, B., Proc. Ind. Acad. Sci. A 12 (1940) 93.

[7] LANDOLT BöRNSTEIN, Numerical data and functional relationships in science and technology (Springer-Verlag) 1979, vol. II, p. 169.
[8] Hand book of Chemistry and Physics, 57e edition (1976-77) p. 248.

[9] McSkimmin, Andreatch, J., Thurston, R., J. Appl. Phys. 36 (1965) 1624.

[10] Ramachandran, G., Chandrasekaran, K., Acta Crystallogr. 10 (1957) 671.

[11] Abrahams, S., Glass, A., Nassau, K., Solid State Commun. 24 (1977) 515. 